\title{
Long-term persistence of donor nuclei in a Duchenne muscular dystrophy patient receiving bone marrow transplantation
}

\author{
Emanuela Gussoni, ${ }^{1}$ Richard R. Bennett, ${ }^{1}$ Kristina R. Muskiewicz, ${ }^{1}$ Todd Meyerrose,${ }^{2}$ \\ Jan A. Nolta, ${ }^{2}$ Irene Gilgoff, James Stein, ${ }^{4}$ Yiu-mo Chan, ${ }^{5}$ Hart G. Lidov, ${ }^{1}$ \\ Carsten G. Bönnemann, ${ }^{6}$ Arpad von Moers, ${ }^{7}$ Glenn E. Morris, ${ }^{8}$ Johan T. den Dunnen, ${ }^{9}$ \\ Jeffrey S. Chamberlain, ${ }^{10}$ Louis M. Kunkel, ${ }^{1,11}$ and Kenneth Weinberg ${ }^{2}$ \\ ${ }^{1}$ Division of Genetics, Children's Hospital, Boston, Massachusetts, USA \\ ${ }^{2}$ Division of Research Immunology and Bone Marrow Transplantation, Department of Pediatrics, \\ Children's Hospital Los Angeles, Los Angeles, California, USA \\ ${ }^{3}$ Department of Pediatrics, Rancho Los Amigos Medical Center, Downey, California, USA \\ ${ }^{4}$ Department of Surgery, Children's Hospital Los Angeles, Los Angeles, California, USA \\ ${ }^{5}$ Geisinger Hospital, Weis Center for Research, Danville, Pennsylvania, USA \\ ${ }^{6}$ Division of Neurology, Children's Hospital of Philadelphia, and University of Pennsylvania, Philadelphia, Pennsylvania, USA \\ ${ }^{7}$ University Children's Hospital, Department of Neuropediatrics, Charité, Berlin, Germany \\ ${ }^{8}$ North East Wales Institute, Wrexham, United Kingdom \\ ${ }^{9}$ Center for Human and Clinical Genetics, Leiden University Medical Center, Leiden, The Netherlands \\ ${ }^{10}$ Department of Neurology, University of Washington, Seattle, Washington, USA \\ ${ }^{11}$ The Howard Hughes Medical Institute at Children's Hospital, Boston, Massachusetts, USA
}

Duchenne muscular dystrophy (DMD) is a severe progressive muscle-wasting disorder caused by mutations in the dystrophin gene. Studies have shown that bone marrow cells transplanted into lethally irradiated $m d x$ mice, the mouse model of DMD, can become part of skeletal muscle myofibers. Whether human marrow cells also have this ability is unknown. Here we report the analysis of muscle biopsies from a DMD patient (DMD-BMT1) who received bone marrow transplantation at age 1 year for $\mathrm{X}$-linked severe combined immune deficiency and who was diagnosed with DMD at age 12 years. Analysis of muscle biopsies from DMD-BMT1 revealed the presence of donor nuclei within a small number of muscle myofibers $(0.5-0.9 \%)$. The majority of the myofibers produce a truncated, in-frame isoform of dystrophin lacking exons 44 and 45 (not wild-type). The presence of bone marrow-derived donor nuclei in the muscle of this patient documents the ability of exogenous human bone marrow cells to fuse into skeletal muscle and persist up to 13 years after transplantation.

This article was published online in advance of the print edition. The date of publication is available from the JCI website, http://www.jci.org. J. Clin. Invest. 110:807-814 (2002). doi:10.1172/JCI200216098.

\section{Introduction}

Bone marrow transplantation (BMT) is used to treat a variety of human diseases such as severe combined immune deficiency (SCID) $(1,2)$, Fanconi anemia $(3,4)$, osteogenesis imperfecta $(5,6)$, and inborn errors of

Received for publication June 5, 2002, and accepted in revised form July 23, 2002.

Address correspondence to: Louis Kunkel, Division of Genetics, Howard Hughes Medical Institute, Children's Hospital, 320 Longwood Avenue, Boston, Massachusetts 02115, USA. Phone: (617) 355-7576; Fax: (617) 277-0496;

E-mail: kunkel@enders.tch.harvard.edu.

Conflict of interest: No conflict of interest has been declared. Nonstandard abbreviations used: bone marrow transplantation (BMT); severe combined immune deficiency (SCID); bone marrow (BM); mesenchymal stem cell progenitors (MSCs); Duchenne muscular dystrophy (DMD); graft-versus-host disease (GVHD); fluorescence in situ hybridization (FISH);

4',6-diamidine-2-phenylindole dihydrochloride (DAPI). metabolism or macrophage function such as lysosomal storage diseases or osteopetrosis $(7,8)$. Adult bone marrow (BM) contains mesenchymal stem cell progenitors (MSCs), which can give rise to osteocytes, chondrocytes, adipocytes, and skeletal muscle $(9,10)$. Marrow also contains progenitors for neural $(11,12)$, hepatic $(13,14)$, cardiac $(15,16)$, and epithelial cells (17), indicating the potential of BMT to repair nonhematopoietic tissues. Engraftment of donor-derived cells in nonhematopoietic tissues has been described in human BMT recipients $(18,19)$. However, recent findings demonstrated that BM cells fuse to and subsequently adapt the phenotype of host cells in vitro, thus questioning the transdifferentiation abilities of BM cells in vivo (20).

Duchenne muscular dystrophy (DMD), caused by mutation of the dystrophin gene, is the most common and severe form of muscular dystrophy, with loss of independent ambulation occurring at approximately 10 
years of age $(21,22)$. Loss of dystrophin protein is also observed in the $m d x$ mouse, the mouse model of DMD (23). In $m d x$ mice and DMD patients, dystrophin expression is restored within revertant myofibers and results from skipping of exons surrounding the mutation, giving rise to truncated in-frame transcripts (24-26).

Skeletal muscle myofibers are multinucleate syncytial structures that arise from fusion of mononuclear myoblasts (27). Besides myoblasts, other cells can participate in the formation of myotubes in vitro (28) or fuse to preexisting myofibers in vivo (29-33). Thus, for therapy of muscle disorders, fusion of wild-type cells to dystrophic myofibers leads to the incorporation of normal nuclei capable of expressing the desired gene product (34). Studies in the $m d x$ mouse have indicated that different cell types can contribute to the repair of dystrophic myofibers in vivo, including myoblasts (35), fibroblasts (29), and BM cells after BMT (30-32). Thus, preclinical testing of BMT for therapy of muscular dystrophy is currently ongoing. Here we describe a patient, DMD-BMT1, who received BMT at 1 year of age for X-linked SCID, was diagnosed with DMD at age 12 years, and has a very mild muscular dystrophy phenotype. This fortuitous case represents a unique opportunity to study whether human bone marrow cells have the potential to engraft into diseased skeletal muscle, persist for 13 years after BMT, and contribute to the mild phenotype of the patient.

\section{Methods}

The studies were performed in accordance with a protocol approved by the Committee on Clinical Investigations (Institutional Review Board) of Children's Hospital Los Angeles, with the informed consent of the patient's parents and the assent of the patient, referred to as DMD-BMT1.

Analysis of skin, marrow, and peripheral blood DNA samples. DMD-BMT1 skin fibroblasts and BM-derived MSCs were grown to confluence in DMEM 10\% FCS or Dexter's Original Medium (Life Technologies Inc., Grand Island, New York, USA). Peripheral blood lymphocytes were isolated by Ficoll separation (Amersham-Pharmacia Biotech Europe GmbH, Uppsala, Sweden), and FACS sorting was used to purify $\mathrm{CD}^{+} \mathrm{T}$ lymphocytes and $\mathrm{CD} 13 / \mathrm{CD} 14^{+}$ myeloid cells, respectively. Genomic DNA was extracted using Puregene kit (Gentra Systems Inc., Minneapolis, Minnesota, USA). Dystrophin exons were amplified using a comprehensive set of primers (36). For each PCR reaction, $300 \mathrm{ng}$ of forward or reverse primers were added to $200 \mathrm{ng}$ of template genomic DNA. The sequences of exon 45 primers were as follows: 45F2 5 '-GGTGTCTTTCTGTCTTGTATCCTT-3'; 45R2 5'-CCTATTAGATCTGTCGCCCTAC-3'. Red Hot Taq (ABgene North America, Rochester, New York, USA) was used at $1.5 \mathrm{U}$ per reaction with $1.5 \mathrm{mM} \mathrm{MgCl}_{2}$. Genomic DNA was initially denatured at $94^{\circ} \mathrm{C}$ for 3 minutes. Amplification was performed for 35 cycles under the following conditions: $94^{\circ} \mathrm{C}$ for 5 seconds, $50-56^{\circ} \mathrm{C}$ (depending on the primer pair) for 15 seconds, and $72^{\circ} \mathrm{C}$ for 30 seconds, with a single, final extension at $72^{\circ} \mathrm{C}$ lasting 3 minutes. PCR product sequencing was performed on an ABI 373 fluorescent sequencer after purification using QIAquick PCR purification kit (QIAGEN Inc., Valencia, California, USA).

RT-PCR analysis of dystrophin transcripts. Total RNA was extracted using the RNeasy kit (QIAGEN Inc.), and cDNA was synthesized and amplified with the One Step RT-PCR kit (QIAGEN Inc.). Forward and reverse primers were as follows: CC42F-C48R (CC42F 5'-ATGCTCCTGACCTCTGTGCTAAG-3'; C48R 5'-ACCACAGCAGCAGATGATTTAACT-3') or CC42F-CC45R (CC45R 5'-GGCTTCCCAATTTTTCCTGTAGAA-3') or CC45F-CC47R (CC45F 5'-CAATCCTCAAAAACAGATGCCAGT-3'; C47R 5'-TTCTGGGCTTATGGGAGCACTTAC$\left.3^{\prime}\right)$. Reverse transcription was performed in a Hybaid thermocycler for 30 minutes at $50^{\circ} \mathrm{C}$ followed by 15 minutes at $95^{\circ} \mathrm{C}$. Amplification was performed for 30 cycles under the following conditions: $94^{\circ} \mathrm{C}$ for 1 minute, $52^{\circ} \mathrm{C}$ for 40 seconds, and $72^{\circ} \mathrm{C}$ for 50 seconds, with a final extension at $72^{\circ} \mathrm{C}$ for 10 minutes. A second round of PCR amplification was performed using $5 \mu \mathrm{l}$ of undiluted (DMD-BMT1) or diluted (1:100, unaffected control) PCR product from the first round of amplification. Two hundred nanograms of dystrophin primers were used (C43F-C48R; C43F-C47R; C43F-CC45R; CC45F-C46R) (C43F 5'-CGCCTGTGGAAAGGGTGAAGCTAC-3'; C46R 5'-TTCAAGTGGGATACTAGCAATGT-3') under identical amplification conditions. PCR products were concentrated in a speed vacuum and loaded on a $1.5 \%$ NuSieve agarose gel. Bands were gel purified (QIAGEN Inc.) and sequenced on an $\mathrm{ABI} 373$ sequencer.

For real-time RT-PCR (Taqman; Applied Biosystems Inc., Foster City, California, USA), primers and probes were designed (ABI Software Inc., Foster City, California, USA) to specifically amplify full-length dystrophin (primers 44F 5'-TCCCCAGTTGCATTCAATGTT-3'; 45R $5^{\prime}$-ATCTTAAGGAACTCCAGGATGGC-3'; Taqman probe 5'-TGACAACAGTTTGCCGCTGCCC-3'); 44-46 isoform (out of frame, primers 44F $5^{\prime}$-TGACAAGATATTCTTTTGTTCTTCTAGCCT-3'; 46R 5'-CAGTTTCTCAGAAAGACACAAATTCC-3'; Taqman probe 5'-AAGATACCATTTGTATTTAGCATGTTCCCAATTCTCA-3'); 43 -46 (in frame, primers 43F 5'-TCATTTAAATCTCTTTGAAATTCTGACAA-3'; 46R 5'-CCAATGGGAAAAAGTTAACAAAATGTA-3'; Taqman probe $5^{\prime}$-TCTTTTGTTCTTCTAGCCCTTGTCGGTCCTT- $3^{\prime}$ ), and $44-48$ (in frame, primers 44F $5^{\prime}$-CATGCTAAATACAAATGGTATCTTAAGGT-3'; 48R 5'-CAAGCTGCCCAAGGTCTTTTAT-3'; Taqman probe 5'-TCCAGAGCTTTACCTGAGAAACAAGGAGAAATTG-3'). Primers and probes were used in the Taqman Universal PCR master mix at a final concentration of $200 \mu \mathrm{M}$. PCR conditions were $2 \mathrm{~min}$ utes at $50^{\circ} \mathrm{C}$ (once), 10 minutes at $95^{\circ} \mathrm{C}$ (once) followed by 15 seconds at $95^{\circ} \mathrm{C}$, and 1 minute at $60^{\circ} \mathrm{C}$ for 40 cycles (single-round PCR).

Immunohistochemistry. DMD-BMT1 MSCs grown on glass slide chambers were stained with mouse antihuman CD105 (dilution 1:20; BD PharMingen, San Diego, California, USA). For muscle tissue, $10-\mu \mathrm{m}$ sections were cut from snap-frozen muscle biopsies. Dystrophin was detected with the following primary Ab's: 
MANDYS102 (exon 43 epitope), MANEX 46B (exon 46 epitope) (24,37), diluted 1:20; NCL-DYS1 and NCLDYS2 (Novocastra Laboratories, Newcastle, United Kingdom), diluted 1:100. The affinity-purified rabbit polyclonal Ab CAP 6-10 directed to the dystrophin cDNA residues 6,181-9,544 (exon 42-64 protein epitopes) was used at a final concentration of $0.3 \mu \mathrm{g} / \mathrm{ml}$ (diluted 1:1,000) (38). Cultured cells or tissue sections were incubated with the primary $\mathrm{Ab}$ overnight at $4^{\circ} \mathrm{C}$, washed three times for 10 minutes in PBS at room temperature, and incubated at room temperature for 1 hour with either a FITC- or a Cy5-conjugated secondary $\mathrm{Ab}$ (diluted 1:100; Jackson Immunochemicals Inc., West Grove, Pennsylvania, USA). Sections were washed again in PBS, mounted in Vectashield (Vector Laboratories, Burlingame, California, USA), and examined using a Zeiss Axiophot microscope (Carl Zeiss Inc., Thornwood, New York, USA) (32). The same protocol was followed for simultaneous detection of dystrophin-positive myofibers and $\mathrm{T}$ cells using anti-dystrophin CAP 6-10 (diluted 1:1,000) and anti-human CD2 (diluted 1:100; DAKO Corp., Carpinteria, California, USA) overnight at $4^{\circ} \mathrm{C}$, followed by incubations with anti-rabbit Cy 5 and anti-mouse FITC (Jackson Immunochemicals Inc.), both diluted 1:100. For flow cytometry, anti-CD5 (Leu-1) antibody was purchased from Becton-Dickinson Immunocytometry Systems (San Jose, California, USA).

Fluorescence in-situ bybridization. Following immunohistochemistry, coverslips were removed, and muscle tissue sections were refixed in Histochoice (AMRESCO Inc., Solon, Ohio, USA) as described previously (39). The genomic phage clone $\lambda \mathrm{NN}$. 1 or the cosmid clone cyD4.87 (40) containing genomic sequences flanking and including dystrophin exon 45 were separately labeled by nick translation with digoxigenin-11-dUTP (Roche Molecular Biochemicals, Indianapolis, Indiana, USA) using $1 \mu \mathrm{g}$ of purified DNA (41). The X chromosome FITC-labeled centromeric probe was purchased (Rainbow Scientific Inc., Windsor, Connecticut, USA) and used according to the manufacturer's instructions. Sections and probe were denatured at $70^{\circ} \mathrm{C}$, and $200 \mathrm{ng}$ of denatured, preannealed digoxigenin-labeled $\lambda$ NN.1 or CyD4.87 probe was added per slide. Sections were hybridized overnight at $37^{\circ} \mathrm{C}$ and processed as described previously (39).

\section{Results}

Clinical history. DMD-BMT1 is a 15-year-old male who was diagnosed with X-linked SCID at 6 months. There was a maternal family history of male infants who had died of opportunistic infections in infancy. DMD-BMT1 received conditioning with cyclophosphamide and anti-thymocyte globulin prior to receiving an HLA-haploidentical $\mathrm{T}$ cell-depleted BMT from his father. T cell depletion was accomplished by incubation of the donor marrow with anti-CD5 mAb and complement. Because the patient did not receive myeloablative conditioning, the expected outcome after BMT is that he would have donor-derived T lymphocytes, but that all other hematopoietic lineages would be host derived. After transplant, DMD-BMT1 had grade 3 acute graft-versus-host disease (GVHD), which required immunosuppression with cyclosporine and steroids. DMD-BMT1's immune system has shown persistent absence of humoral function, manifested by chronic recurrent middle ear infections. He has been treated with intravenous immunoglobin monthly for his hypogammaglobulinemia.

DMD-BMT1 walked at 2 years of age, the delay being attributed to his difficult post-BMT course. His parents reported that he had no interest in physical activities, but his intellectual development has been above average. During a scheduled visit at age 12 , findings consistent with muscular dystrophy were noted, including elevated serum creatine kinase value $(8,000-9,000 \mathrm{IU} / \mathrm{ml})$, history of falling at school, shortened Achilles tendons, limb muscle hypertrophy, and Gower's sign (22). Analysis of blood leukocyte DNA revealed a deletion of dystrophin exon 45 , although a faint signal was present after long exposure of the autoradiogram. Currently, at age 15 years, DMD-BMT1 can still walk, but uses a wheelchair approximately $50 \%$ of the time.

Molecular diagnosis of DMD-BMT1. To confirm the deletion of dystrophin exon 45 in DMD-BMT1, a skin fibroblast cell line was established from the patient. PCR amplification of the fibroblast-derived DNA was performed using primers to all 79 dystrophin exons. All exons amplified from an unaffected control, and only exon 45 failed to amplify from DMD-BMT1, thus agreeing with the original finding that the deletion is restricted to dystrophin exon 45 (Figure 1a).

To determine the contribution of donor cells to the peripheral blood of DMD-BMT1 after BMT, genomic DNA was extracted from DMD-BMT1's T lymphocyte and myeloid cell fractions. Amplification by PCR using primers to dystrophin exons 40 and 45 revealed that the $\mathrm{T}$ lymphocytes contained cells of donor origin as expected, while only a very faint exon 45 band was seen in the myeloid cells, indicating their predominant host origin (Figure 1b). Amplification of exon 40 was unaltered in all fractions.

To exclude the possibility that DMD-BMT1 is a somatic mosaic for a dystrophin gene deletion, which would limit the ability to unequivocally detect donor-derived cells, his mother's carrier status was ascertained by two independent methods. PCR amplification and sequence analysis of dystrophin exon 45 on peripheral blood-derived genomic DNA of DMD-BMT1 (T lymphocytes and myeloid cells) and his parents revealed a polymorphism 143 bases into the intron flanking exon 45 . The mother was apparently homozygous for G, while the father and DMD-BMT1 had an A in this position (Figure 1c). Because DMD is an $\mathrm{X}$-linked disorder, the absence of a $\mathrm{G}$ in the myeloid cells from DMD-BMT1 is consistent with the inheritance of a null DMD allele from the mother. Her DMD carrier status was further confirmed by fluorescence in situ hybridization (FISH) analysis using a genomic DNA probe containing dystrophin exon 45 ( $\lambda \mathrm{NN}$.1). Chromosome spreads from an unaffected female showed the presence of hybridization signals on the short arm of 
$\mathbf{a}$

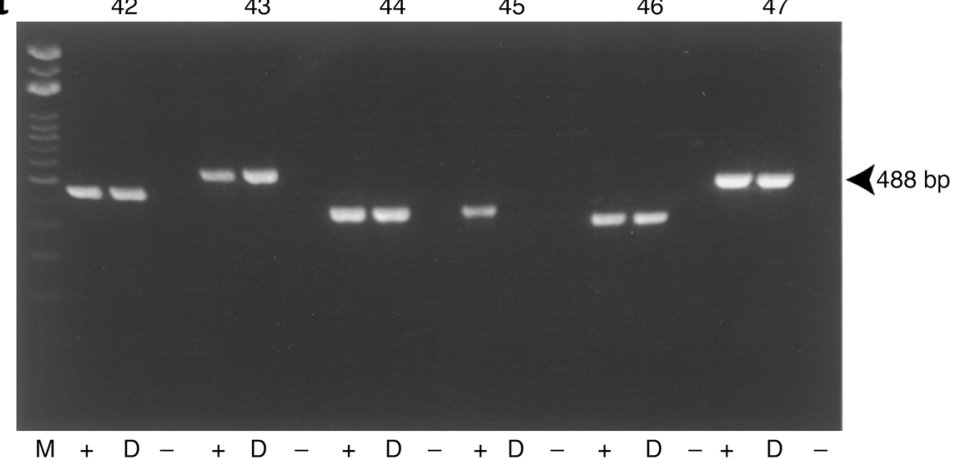

C b

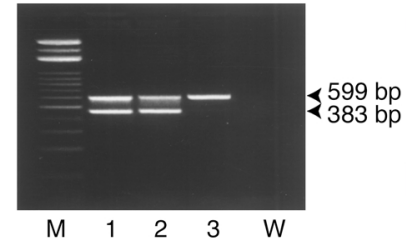

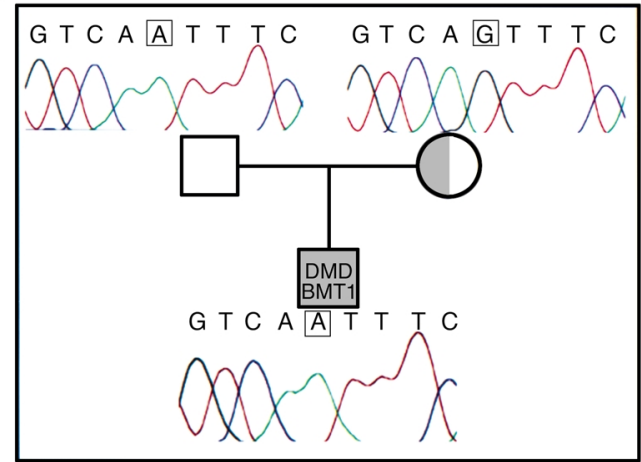

\section{Figure 1}

Deletion of dystrophin exon 45 in DMD-BMT1 genomic DNA by PCR. (a) DNA amplification performed using primers to dystrophin exons 42-47. Genomic DNA samples are from an unaffected individual (+) or DMD-BMT1 skin fibroblasts (D). The negative water control (-) is present for each primer set. M, molecular weight markers. (b) Coamplification of dystrophin exons 40 (599 bp) and 45 (383 bp) from genomic DNA of an unaffected individual (lane 1), DMD-BMT1 purified T cells (lane 2), or myeloid cells (lane 3). W, water control. (c) Chromatograms of the DNA sequence analyses performed on the dystrophin exon 45 band amplified by PCR from DMD-BMT1 and his parents. DMD BMT-1 and his father are homozygous for $A$ at nucleotide 143 upstream of exon 45, while the mother has a $G$ in this position.

both $\mathrm{X}$ chromosomes (Figure 2a), while chromosome spreads from DMD-BMT1's mother consistently showed signals on only one $\mathrm{X}$ chromosome (Figure $2 \mathrm{~b}$ ), proving her carrier status and essentially excluding the possibility that DMD-BMT1 was a somatic mosaic for dystrophin exon 45 deletion. Interphase nuclei from DMDBMT1-cultured fibroblasts showed no hybridization with the dystrophin exon 45 probe, while an $\mathrm{X}$ chromosome centromeric probe consistently showed one hybridization signal (Figure 2c).

To evaluate whether MSCs of donor origin were still present in DMD-BMT1 13 years after transplantation, a marrow aspirate was performed and the adherent cells were grown in vitro. The MSC culture was greater than $99 \%$ positive for CD105, a MSC marker $(10,42)$ (Figure 2d). Simultaneous FISH and immunofluorescence analyses demonstrated the presence of donor nuclei within $\mathrm{CD} 105^{+} \mathrm{MSCs}$ at a frequency of 9 in 1,165 nuclei $(0.77 \%)$ (Figure 2e). Recent reports have indicated that mouse BM cells can fuse spontaneously to other mononuclear cell types and subsequently convert to a large tetraploid cell upon nuclear fusion (20). To determine if the donor cells in DMD-BMT1 MSC cultures were diploid and not fused to host cells we examined interphase nuclei by FISH using an X chromosome centromeric probe (green) and a dystrophin exon 45 DNA probe (red), simultaneously. Donor nuclei appeared to have single red and green hybridization signals (Figure $2, \mathrm{f}-\mathrm{g}$ ), while nuclei from an unaffected female revealed the presence of double green and red hybridization signals in greater than $90 \%$ of the nuclei, as expected (Figure $2, \mathrm{~h}-\mathrm{i})$. These results indicate that donor-derived MSC nuclei present in DMD-BMT1 marrow over a decade after BMT are diploid, not fused with host nuclei.

Muscle biopsy analyses. To ascertain engraftment of donor cells and expression of normal dystrophin in DMD-BMT1's skeletal muscle, biopsies were surgically removed from the left calf and right quadriceps. Muscle biopsies from an unaffected individual, as well as two unrelated DMD patients (D1 and D2), were performed for clinical indications independent of this study and were provided from other institutions as control tissues. DMD patients D1 and D2 had a confirmed single-exon deletion of dystrophin exon 45 , but did not receive BMT (data not shown).

Immunofluorescence and FISH analyses. For immunofluorescence analysis, frozen muscle biopsies from DMDBMT1 and from control DMD patients D1 and D2 were serially sectioned and individually incubated with Ab's recognizing different dystrophin epitopes. Upon 


\section{Figure 2}

Detection of dystrophin exon 45 deletion in DMD-BMT1 and his mother by FISH analysis. (a) Unaffected female. Chromosomes are counterstained in blue by 4',6-diamidine-2-phenylindole dihydrochloride (DAPI) and both $X$ chromosomes have red hybridizations signals using a dystrophin exon 45-specific probe. (b) DMD-BMT1's mother's chromosome spreads. Only one X chromosome hybridized with the dystrophin exon 45-specific probe, thus confirming her DMD carrier status for dystrophin exon 45 deletion. (c) DMD-BMT1 fibroblast nuclei hybridized with an X-centromeric probe (green) and a dystrophin exon 45 probe (red), which failed to hybridize. ( $\mathbf{d}$ and $\mathbf{e}$ ) Detection of donor-derived nuclei in MSC cultures of DMD-BMT1. (d) By immunofluorescence, more than $95 \%$ of cultured MSCs from DMDBMT1 are positive for CD105 (green). (e) FISH analysis using a dystrophin exon 45 cosmid probe (red) shows presence of donor-derived MSCs. ( $\mathbf{f}$ and $\mathbf{g}$ ) Simultaneous hybridization of DMD-BMT1 MSC nuclei with an $\mathrm{X}$-centromeric DNA probe (green) and dystrophin exon 45 probe (red) by FISH. Donor nuclei in DMD-BMT1 MSCs are diploid. ( $h$ and $\mathbf{i}$ ) Nuclei from an unaffected female hybridized by FISH with the X-centromeric and dystrophin exon $45 \mathrm{FISH}$ probes. Two green and red hybridization signals are expected in each nucleus. Nuclei are counterstained in blue by DAPI.
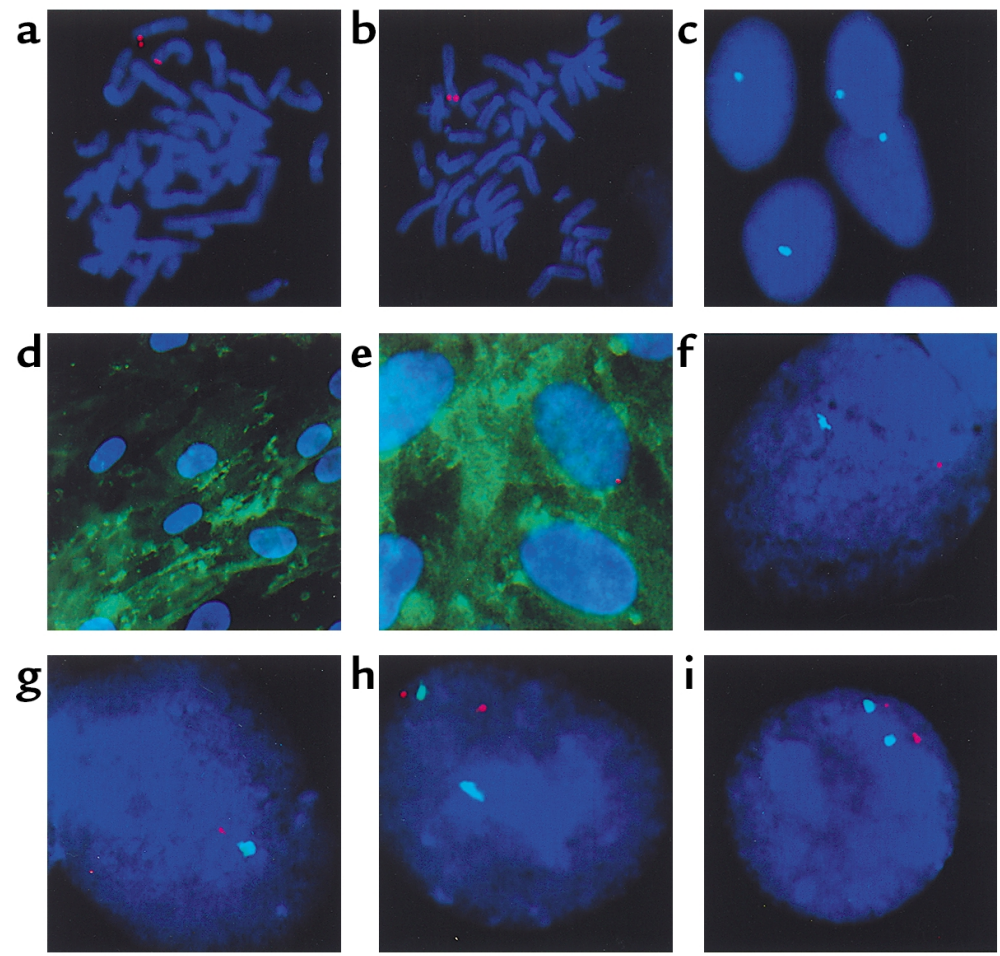

examination, clusters of dystrophin-positive myofibers were observed in all patients, with no apparent difference between sections from DMD-BMT1 (Figure 3, a-c) and nontransplanted DMD patient D1 (Figure 3, $\mathrm{d}-\mathrm{f}$ ) and D2 (data not shown). Sections stained with MANDYS102 recognizing a protein epitope within dystrophin exon 43 $(43,44)$ (Figure 3 , a and d) or CAP6-10 recognizing protein epitopes within dystrophin exons 42-64 (38) (Figure $3, \mathrm{c}$ and $\mathrm{f}$ ) revealed faint dystrophin reactivity in most myofibers and strong reactivity in interspersed, clustered myofibers that also appeared to be positive with MANEX46B, an Ab specific to a protein epitope within dystrophin exon 46 (Figure $3, \mathrm{~b}$ and $\mathrm{e}$ ). There was no detectable difference in dystrophin staining between nontransplanted controls and DMD-BMT1.

FISH analysis of immunostained DMD-BMT1 tissue sections using simultaneously a dystrophin exon 45 DNA probe (red) and a X chromosome centromeric probe (green) revealed the presence of diploid donor nuclei fused within dystrophin-positive myofibers (magenta) with single green and red hybridization signals (Figure 3, g and $h$ ). Tissue sections from an unaffected female revealed the expected double green and red hybridization signals in $63 \%$ of nuclei (Figure $3 i$ ). FISH analysis on muscle tissue sections from D1 or D2 failed to detect dystrophin exon 45 (data not shown).

\section{Figure 3}

Immunohistochemistry/FISH analysis of serial sections from muscle biopsies of DMDBMT1 (a-c) and D1 (d-f). Numbers in panels a-c identify specific myofibers in serial sections; 7 and $7^{\prime}$ represent a split myofiber. (a-f) Tissue sections stained with different dystrophin Ab's show the presence of dystrophin-positive myofibers (green signal). (g-i) Four-color FISH/immunohistochemistry analysis for detection of fused diploid donor nuclei in DMD-BMT1 muscle tissue sections. DMD-BMT1 ( $\mathbf{g}$ and $\mathbf{h}$ ) and unaffected control female (i) tissue sections stained for dystrophin (magenta) and then hybridized by FISH with an X-centromeric probe (green) and a dystrophin exon 45 probe (red). Nuclei are counterstained in blue by DAPI. ex, exon. a
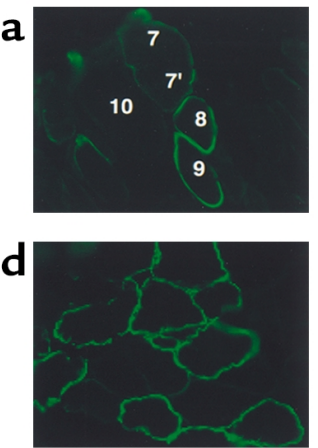

MANDYS102 (ex 43)

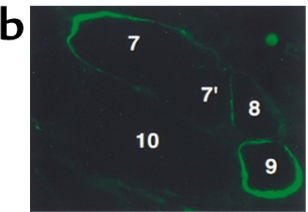

e
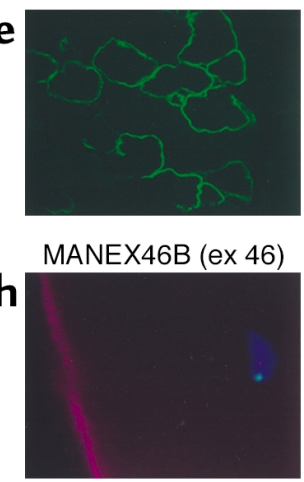

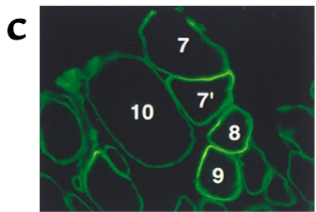

DMD-BMT1

$f$

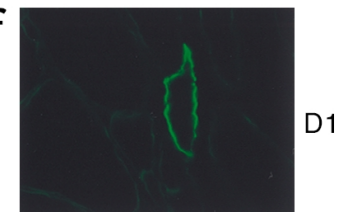

CAP6-10 (ex 42-64)
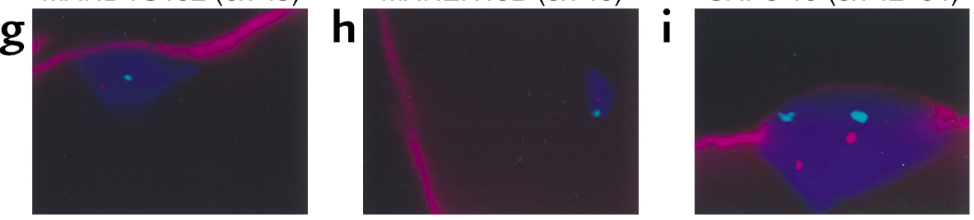


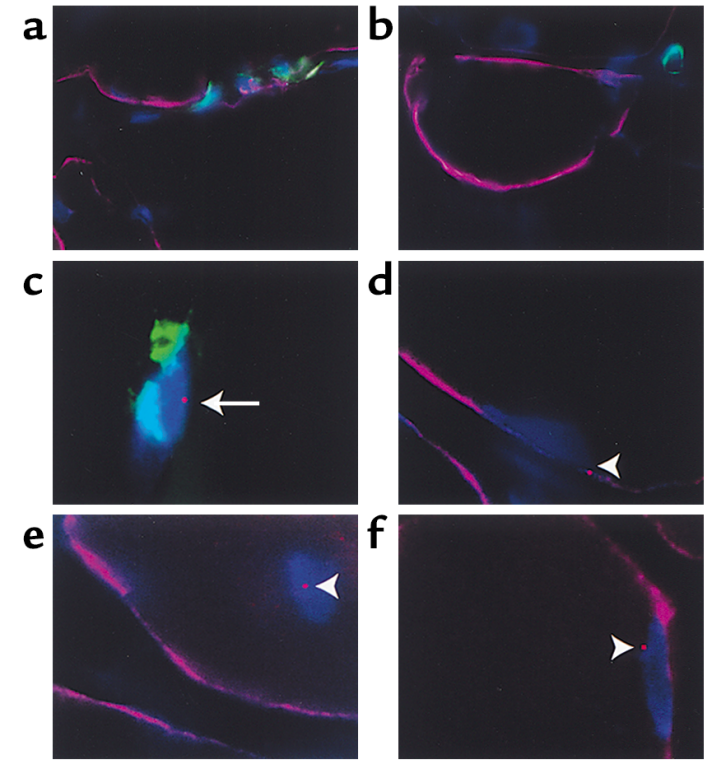

Figure 4

Four-color FISH/immunohistochemistry analyses for codetection of donor-derived nuclei, T cells, and dystrophin-positive myofibers in DMD-BMT1 muscle. T cells are stained in green (FITC), dystrophinpositive myofibers are stained in magenta (Cy5), nuclei are stained in blue (DAPI), and donor nuclei have a red hybridization signal (rhodamine). ( $\mathbf{a}$ and $\mathbf{b}) \mathrm{T}$ cells are not fused to dystrophin-positive myofibers, but appear to reside between myofibers. (c) T cells are of donor origin, as shown by the red dystrophin exon 45 hybridization signal after FISH analysis (white arrow) (d-f) Donor nuclei fused to dystrophin-positive myofibers are not $\mathrm{T}$ cells (white arrowheads).

Since DMD-BMT1 is a hematopoietic chimera in which the T and NK lymphocytes are of donor origin, it was important to establish whether the donor-derived nuclei detected within dystrophin-positive myofibers were due to artifactual overlay of $\mathrm{T}$ lymphocytes or incorporation of donor nuclei in a host myofiber. Simultaneous immunostaining of dystrophin and the $\mathrm{T}$ cell-specific protein CD2 did not show evidence that donor T lymphocytes had fused with the host myofibers. Instead, T lymphocytes were detected only interstitially in two separate series of serial sections spanning a total length of $700 \mu \mathrm{m}$ (Figure 4, a and b). FISH analysis of 11 of these consecutive sections using a dystrophin exon 45 cosmid probe revealed eight interstitial $\mathrm{T}$ lymphocytes of donor origin (Figure 4c), eight donor nuclei fused within dystrophin-positive myofibers lacking the $\mathrm{T}$ lymphocyte marker CD2 (Figure 4, d-f), and two donor nuclei fused in dystrophin-negative myofibers.

$R T$-PCR analyses of $m R N A$ transcripts. To investigate at the molecular level the type(s) of dystrophin transcripts expressed in the muscle of DMD-BMT1, nested RT-PCR analyses were performed. First-round cDNA amplification using primers specific for dystrophin exons 42-48 revealed the presence of a band of predicted size from an unaffected control and a band of reduced size from DMD-BMT1, D1, and D2 (data not shown). Reamplification of diluted PCR products using the nested primers
C43F-C48R or C43F-C47R revealed the presence of multiple bands (Figure 5a), which were purified and sequenced. The largest band corresponded to the transcript lacking exon 45 (out of frame), while the smaller bands were consistent with transcripts either lacking exons 44 and 45 or exons 45, 46, and 47, respectively (Figure $5 \mathrm{~b}$ ). These transcripts are predicted to express inframe, truncated dystrophin proteins 43-46 and 44-48 $(24,45)$. Multiple faint bands were also detected near the expected molecular weight of full-length dystrophin in DMD-BMT1 (Figure 5a). These bands were also purified, but sequence analysis failed to determine whether these transcripts corresponded to full-length dystrophin.

To detect wild-type dystrophin transcripts in DMDBMT1, a first round of PCR amplification was performed using primers CC42F-CC45R or CC45F-C47R, followed by nested PCR amplification using primers C43F-CC45R or CC45F-C46R. Bands of expected size containing exon 45 were amplified from the unaffected control and DMD-BMT1 (Figure 5c). In contrast, amplification of cDNA from D1 and D2 using primers CC42F-CC45R failed to amplify a band, confirming that dystrophin exon 45 is deleted in these patients (data not shown).

To estimate the relative amounts of dystrophin transcripts present in DMD-BMT1 muscle, we performed real-time quantitative RT-PCR analysis (Figure $5 \mathrm{~d}$ ). The most abundant dystrophin cDNA in DMD-BMT1's muscle is the exon 45-deleted isoform, followed by the 43-46 and 44-48 truncated in-frame isoforms (Figure $5 \mathrm{~d})$. There is detectable full-length dystrophin in DMD-BMT1 at an estimated amount of $0.0005 \%$ relative to normal (Figure $5 \mathrm{~d}$ ). A similar amount of normal dystrophin transcripts was detected in cDNA derived from peripheral blood lymphocytes of an unaffected individual (data not shown), thus making it difficult to rule out whether wild-type dystrophin transcripts in DMD-BMT1 are produced by fused donor nuclei or by interstitial donor-derived T lymphocytes. No wild-type dystrophin transcripts were amplified from D1 or D2 muscle-derived cDNA (data not shown).

\section{Discussion}

Studies in rodents have demonstrated that BM contains progenitors for hematopoietic and nonhematopoietic lineages $(9,11,12,13,46)$, including skeletal muscle (30-32). Similarly, human BM cells can give rise to hepatocytes $(18,47,48)$, cardiomyocytes $(19)$, osteocytes $(5$, 6), and epithelial cells in vivo after BMT (18).

DMD-BMT1 has a very mild DMD clinical course, which prompted us to investigate whether the BMT he received for the treatment of X-SCID also slowed the progression of DMD symptoms through fusion of donor cells into dystrophic myofibers, resulting in expression of wild-type dystrophin. Our analyses demonstrate that the relatively mild clinical course of DMD-BMT1 is not likely due to BMT, but to exon skipping and expression of truncated dystrophin protein.

Molecular analyses had indicated that DMD-BMT1 inherited a dystrophin exon 45 deletion from his mother 
a

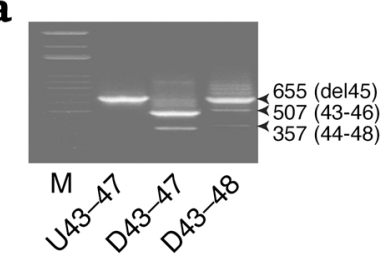

C

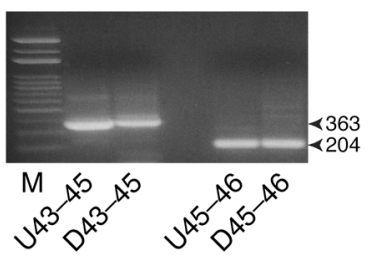

b
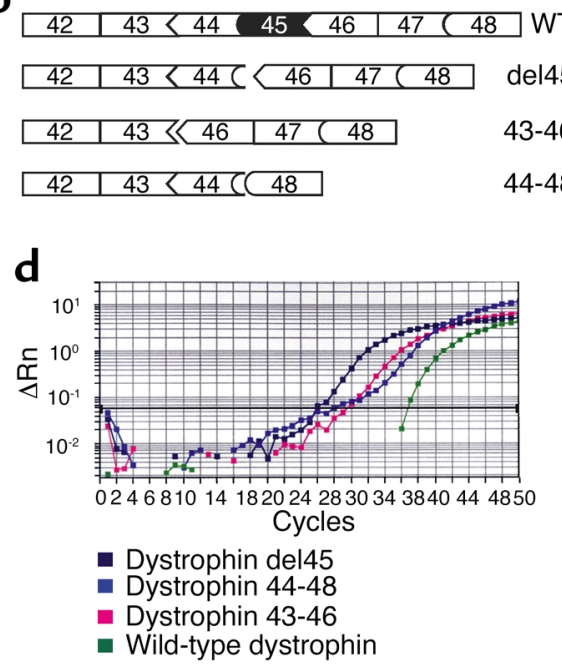

Figure 5

Analysis of dystrophin transcripts expressed in DMD-BMT1's muscle. (a) U43-47 is unaffected control cDNA amplified with primers C43F-C47R; D43-47 and D43-48 are DMD-BMT1's cDNA amplified using primers C43F-C47R or C43F-C48R, respectively. D43-48 shows the presence of three transcripts: del45 (655 bp), 43-46 (507 bp), and 44-48 (357 bp). (b) Schematic diagram of dystrophin transcripts amplified by RT-PCR and sequenced in DMD-BMT1. WT, wild type. (c) Amplification of full-length, donorderived dystrophin in DMD-BMT1 (D43-45, D45-46) and in an unaffected control (U43-45, U45-46). (d) Quantitative RT-PCR analysis on total cDNA derived from DMD-BMT1 skeletal muscle. The cDNA was amplified using primers specific for the 45del isoform (dark blue), 44-48 in-frame truncated dystrophin (light blue), 43-46 inframe truncated dystrophin (pink), and wild-type dystrophin (green). $\Delta \mathrm{Rn}, \Delta$ reaction.

and that he is not a somatic mosaic for the deletion. Thus, FISH analysis using dystrophin exon 45 probes would unequivocally detect the presence of donorderived nuclei in tissues obtained from DMD-BMT1. In contrast, an X chromosome centromeric probe would detect donor and host-derived nuclei. FISH analysis of muscle biopsies from DMD-BMT1 revealed that donorderived nuclei had fused to dystrophin-positive myofibers. These nuclei were diploid and did not become tetraploid in vivo by fusion with host nuclei (20).

Codetection of dystrophin exon 45 by FISH and the T cell-specific marker $\mathrm{CD} 2$ demonstrated that most of the $\mathrm{T}$ cells within the muscle of DMD-BMT1 are of donor origin, as expected from the BMT. This analysis also suggests that donor nuclei fused within dystrophin-positive myofibers are unlikely to be incidental $\mathrm{T}$ cells lying on the host myofiber since they were not expressing CD2. However, these results do not completely exclude the possibility that a donor-derived $\mathrm{T}$ lymphocyte fused to a host myofiber had subsequently downregulated the expression of CD2. The presence of fused nuclei that are derived from the transplanted donor BM cells suggests the existence within adult human $\mathrm{BM}$ of a precursor cell that has the potential to fuse into mature skeletal muscle in vivo. Persistence of donor cells was also detected in MSC cultures 13 years after BMT.

Protein analysis by immunofluorescence revealed that $0.2-0.5 \%$ of DMD-BMT1's myofibers brightly stain using a panel of different dystrophin Ab's. A similar percentage of dystrophin-positive myofibers was also detected in two other DMD patients who had not received $B M T$, suggesting that the $B M$ transplant in DMD-BMT1 did not significantly increase the proportion of dystrophin-positive myofibers. Quantitative RT-PCR analysis of dystrophin transcripts expressed in DMD-BMT1's muscle indicated that the most abundant in-frame dystrophin transcript is $43-46$, followed by $44-48$, and then full-length dystrophin. In light of this, it is not possible to conclude that the mild disease course observed in DMD-BMT1 is due to the presence of the full-length dystrophin derived from donor cells, but is more likely the result of high levels of truncated dystrophin. Other reports have described expression of truncated dystrophin isoforms in DMD patients deleted for dystrophin exon 45 , although the resulting phenotype varies from mild (49-54) to extremely severe (45). Since the first analysis of DMD-BMT1's muscle was performed 13 years after BMT, it remains unclear whether at earlier time points a higher proportion of donor BM cells had engrafted into host myofibers and produced full-length dystrophin.

Because the aim of the haploidentical BM transplant for DMD-BMT1 was treatment of X-SCID, the conditions for transplantation may not have favored donor cell engraftment into host muscle. For example, to achieve engraftment in DMD-BMT1 marrow without inducing fatal GVHD, purging of the donor marrow with an anti-CD5 Ab and complement was used to remove mature $\mathrm{T}$ lymphocytes, which may have resulted in nonspecific losses of myogenic precursors. Conversely, the engraftment that was observed may reflect the profound defect in cell-mediated immunity due to SCID. Previous analyses of long-term engraftment of donor-derived muscle progenitors have used immunodeficient hosts $(30,32)$ and the role of immune responsiveness in determining the ability of marrowderived muscle progenitors to engraft recipient skeletal muscle remains to be characterized.

In summary, this study has demonstrated that adult human-derived BM cells can become part of skeletal muscle myofibers. Donor-derived nuclei were identified in two separate muscle biopsies of a DMD patient 13 years after BMT. The patient described here is the result of the occurrence of DMD in a patient undergoing BMT for an established indication. The application of BMT for the treatment of DMD will require extensive preclinical studies to establish ideal progenitor cells and conditions for transplantation. 


\section{Acknowledgments}

The authors would like to thank Kristine O'Brien for critical review of this manuscript and helpful discussions. Neena Kapoor, Ami Shah, Robertson Parkman, Donald Kohn, and Gay Crooks provided invaluable help in both the care of DMD-BMT1 and the clinical studies described in this paper. The authors are grateful to DMD-BMT1 and his family for their interest, patience, and willingness to participate in the studies. This work was supported by grants from the Charles Hood Foundation (E. Gussoni), the Muscular Dystrophy Association, USA (E. Gussoni, L.M. Kunkel), and the NIH (P50 HL-54850, J.A. Nolta, K. Weinberg; M01 RR00043, K. Weinberg; 1PO1NS4082801, L.M. Kunkel, E. Gussoni). L.M. Kunkel is an Investigator of the Howard Hughes Medical Institute. The clinical care of the patient was provided at the Children's Hospital Los Angeles satellite of the General Clinical Research Center at the University of California.

1. Gatti, R.A., Meuwissen, H.J., Allen, H.D., Hong, R., and Good, R.A. 1968. Immunological reconstitution of sex-linked lymphopenic immunological deficiency. Lancet. 2:1366-1369.

2. Buckley, R.H., et al. 1999. Hematopoietic stem-cell transplantation for the treatment of severe combined immunodeficiency. N. Engl. J. Med. 340:508-516.

3. Kohli-Kumar, M., et al. 1994. Bone marrow transplantation in Fanconi anemia using matched sibling donors. Blood. 84:2050-2054.

4. Gluckman, E., et al. 1995. Bone marrow transplantation for Fanconi anemia. Blood. 86:2856-2862.

5. Horwitz, E.M., et al. 1999. Transplantability and therapeutic effects of bone marrow-derived mesenchymal cells in children with osteogenesis imperfecta. Nat. Med. 5:309-313.

6. Horwitz, E.M., et al. 2001. Clinical responses to bone marrow transplantation in children with severe osteogenesis imperfecta. Blood. 97:1227-1231.

7. Parkman, R., Crooks, G., Kohn, D.B., Lenarsky, C., and Weinberg, K. 1995. Bone marrow transplantation for metabolic diseases. Cancer Treat. Res. 76:87-96.

8. McMahon, C., et al. 2001. Bone marrow transplantation corrects osteopetrosis in the carbonic anhydrase II deficiency syndrome. Blood. 97:1947-1950

9. Prockop, D.J. 1997. Marrow stromal cells as stem cells for nonhematopoietic tissues. Science. 276:71-74.

10. Pittenger, M.F., et al. 1999. Multilineage potential of adult human mesenchymal stem cells. Science. 284:143-147.

11. Brazelton, T.R., Rossi, F.M., Keshet, G.I., and Blau, H.M. 2000. From marrow to brain: expression of neuronal phenotypes in adult mice. Science. 290:1775-1779.

12. Mezey, E., Chandross, K.J., Harta, G., Maki, R.A., and McKercher, S.R. 2000. Turning blood into brain: cells bearing neuronal antigens generated in vivo from bone marrow. Science. 290:1779-1782.

13. Lagasse, E., et al. 2000. Purified hematopoietic stem cells can differentiate into hepatocytes in vivo. Nat. Med. 6:1229-1234.

14. Theise, N.D., et al. 2000. Derivation of hepatocytes from bone marrow cells in mice after radiation-induced myeloablation. Hepatology. 31:235-240.

15. Kocher, A.A., et al. 2001. Neovascularization of ischemic myocardium by human bone-marrow-derived angioblasts prevents cardiomyocyte apoptosis, reduces remodeling and improves cardiac function. Nat. Med. 7:430-436.

16. Orlic, D., et al. 2001. Bone marrow cells regenerate infarcted myocardium. Nature. 410:701-705.

17. Krause, D.S., et al. 2001. Multi-organ, multi-lineage engraftment by a single bone marrow-derived stem cell. Cell. 105:369-377.

18. Korbling, M., et al. 2002. Hepatocytes and epithelial cells of donor origin in recipients of peripheral-blood stem cells. N. Engl. J. Med. 346:738-746.

19. Quaini, F., et al. 2002. Chimerism of the transplanted heart. N. Engl. J. Med. 346:5-15.

20. Terada, N., et al. 2002. Bone marrow cells adopt the phenotype of other cells by spontaneous cell fusion. Nature. 416:542-545.

21. Emery, A. 1988. Duchenne muscular dystrophy. Oxford University Press. New York, New York, USA.

22. Engel, A.G., Yamamoto, M., and Fischbeck, K.H. 1994. Dystrophinopathies In Myology. 2nd ed. Volume 2. A.G. Engel and C. Franzini-Armstrong, editors. McGraw Hill. New York, New York, USA. 1130-1188.

23. Sicinski, P., et al. 1989. The molecular basis of muscular dystrophy in the $m d x$ mouse: a point mutation. Science. 244:1578-1580
24. Thanh, L.T., Nguyen, T.M., Helliwell, T.R., and Morris, G.E. 1995. Characterization of revertant muscle fibers in Duchenne muscular dystrophy, using exon-specific monoclonal antibodies against dystrophin. Am.J. Hum. Genet. 56:725-731.

25. Wilton, S.D., Dye, D.E., and Laing, N.G. 1997. Dystrophin gene transcripts skipping the $m d x$ mutation. Muscle Nerve. 20:728-734

26. Lu, Q.L., et al. 2000. Massive idiosyncratic exon skipping corrects the nonsense mutation in dystrophic mouse muscle and produces functional revertant fibers by clonal expansion. J. Cell Biol. 148:985-996.

27. Buckingham, M. 2001. Skeletal muscle formation in vertebrates. Curr. Opin. Genet. Dev. 11:440-448.

28. Chaudhari, N., Delay, R., and Beam, K.G. 1989. Restoration of normal function in genetically defective myotubes by spontaneous fusion with fibroblasts. Nature. 341:445-447.

29. Gibson, A.J., et al. 1995. Dermal fibroblasts convert to a myogenic lineage in $m d x$ mouse muscle. J. Cell Sci. 108:207-214.

30. Ferrari, G., et al. 1998. Muscle regeneration by bone marrow-derived myogenic progenitors. Science. 279:1528-1530.

31. Bittner, R.E., et al. 1999. Recruitment of bone-marrow-derived cells by skeletal and cardiac muscle in adult dystrophic $m d x$ mice. Anat. Embryol. (Berl.). 199:391-396.

32. Gussoni, E., et al. 1999. Dystrophin expression in the $m d x$ mouse restored by stem cell transplantation. Nature. 401:390-394.

33. Pye, D., and Watt, D.J. 2001. Dermal fibroblasts participate in the formation of new muscle fibres when implanted into regenerating normal mouse muscle. J. Anat. 198:163-173.

34. Partridge, T.A. 1991. Invited review: myoblast transfer: a possible therapy for inherited myopathies? Muscle Nerve. 14:197-212.

35. Blau, H.M., and Springer, M.L. 1995. Muscle-mediated gene therapy N. Engl.J. Med. 333:1554-1556.

36. Bennett, R.R., Dunnen, J., O’Brien, K.F., Darras, B.T., and Kunkel, L.M 2001. Detection of mutations in the dystrophin gene via automated DHPLC screening and direct sequencing. BMC Genet. 2:17. http://www. biomedcentral.com/1471-2156/2/17.

37. Le Thiet, T., et al. 1995. Characterization of genetic deletions in Becker muscular dystrophy using monoclonal antibodies against a deletion-prone region of dystrophin. Am. J. Med. Genet. 58:177-186.

38. Byers, T.J., Lidov, H.G.W., and Kunkel, L.M. 1993. An alternative dystrophin transcript specific to the peripheral nerve. Nat. Genet. 4:77-80.

39. Gussoni, E., et al. 1996. A method to codetect introduced genes and their products in gene therapy protocols. Nat. Biotechnol. 14:1012-1016.

40. Rosenberg, C., et al. 1998. Clinical diagnosis of heterozygous dystrophin gene deletions by fluorescence in situ hybridization. Neuromuscul. Disord. 8:447-452.

41. Lichter, P., et al. 1989. High-resolution mapping of human chromosome 11 by in situ hybridization with cosmid clones. Science. 247:64-69.

42. Cheifetz, S., et al. 1992. Endoglin is a component of the transforming growth factor-beta receptor system in human endothelial cells. J. Biol. Chem. 267:19027-19030.

43. Nguyen, T.M., Ginjaar, I.B., van Ommen, G.J., and Morris, G.E. 1992. Monoclonal antibodies for dystrophin analysis. Epitope mapping and improved binding to SDS-treated muscle sections. Biochem. J. 288:663-668.

44. Nguyen, T.M., and Morris, G.E. 1993. Use of epitope libraries to identify exon-specific monoclonal antibodies for characterization of altered dystrophins in muscular dystrophy. Am. J. Hum. Genet. 52:1057-1066.

45. Prior, T.W., et al. 1997. Dystrophin expression in a Duchenne muscular dystrophy patient with a frame shift deletion. Neurology. 48:486-488.

46. Woodbury, D., Schwarz, E.J., Prockop, D.J., and Black, I.B. 2000. Adult rat and human bone marrow stromal cells differentiate into neurons. J. Neurosci. Res. 61:364-370.

47. Alison, M.R., et al. 2000. Hepatocytes from non-hepatic adult stem cells. Nature. 406:257.

48. Theise, N.D., et al. 2000. Liver from bone marrow in humans. Hepatology. 32:11-16

49. Baumbach, L.L., Chamberlain, J.S., Ward, P.A., Farwell, N.J., and Caskey, C.T. 1989. Molecular and clinical correlations of deletions leading to Duchenne and Becker muscular dystrophies. Neurology. 39:465-474.

50. Gillard, E.F., et al. 1989. Molecular and phenotypic analysis of patients with deletions within the deletion-rich region of the Duchenne muscular dystrophy (DMD) gene. Am. J. Hum. Genet. 45:507-520.

51. Hodgson, S., et al. 1989. Correlation of clinical and deletion data in Duchenne and Becker muscular dystrophy. J. Med. Genet. 26:682-693.

52. Nicholson, L.V., et al. 1993. Integrated study of 100 patients with Xp21 linked muscular dystrophy using clinical, genetic, immunochemical, and histopathological data. Part 2. Correlations within individual patients. J. Med. Genet. 30:737-744.

53. Winnard, A.V., et al. 1993. Characterization of translational frame exception patients in Duchenne/Becker muscular dystrophy. Hum. Mol. Genet 2:737-744.

54. Bornemann, A., and Anderson, L.V. 2000. Diagnostic protein expression in human muscle biopsies. Brain Pathol. 10:193-214 